\title{
Influence d'un blocage de l'extrémité du pivot d'un semis de chêne, sur la morphogenèse de son système racinaire
}

\author{
M. LAMOND, R. TAVAKOL * et A. RIEDACKER * \\ Université de Clermont-Ferrand, France \\ * I.N.R.A., Centre de Recherches forestières Nancy-Champenoux, F 54280 Seichamps \\ avec la collaboration technique de MM. MULLER, GIORIA \\ et HENRION (pour les dessins)
}

\begin{abstract}
Résumé
La pointe de pivots de semis de chêne (Quercus robur L.) de différentes longueurs $(6 \mathrm{~cm}-20 \mathrm{~cm}-35 \mathrm{~cm})$ élevés en caisson à aérosol nutritif ou sur tourbe en minirhizotron a été bloquée. Cela entraîne dans les deux cas une stimulation des racines secondaires les plus distales et les plus grosses qui, sinon, seraient restées fines, obliques et à croissance peu durable. Certaines poursuivent leur croissance et deviennent orthogéotropes.

Lorsque toute la partie du pivot portant des extrémités en croissance est plâtrée, des racines secondaires qui avaient cessé de s'allonger recommencent à croître. Il apparaît aussi des racines secondaires tardives, dont le diamètre de l'apex est dès le début supérieur aux racines secondaires apparues en séquence normale. Leur croissance sera également plus importante et plus durable que celle de ces dernières. Sur caisson à aérosol, des racines secondaires qui avaient cessé de s'allonger peuvent spontanément recommencer à s'allonger pendant quelques jours sans que le pivot soit bloqué, en même temps qu'apparaissent des racines secondaires tardives. Sur tourbe, on n'observe cela qu'après blocage de la croissance axiale du pivot.
\end{abstract}

Les résultats observés semblent pouvoir s'expliquer en faisant appel à la fois :

1) à des phénomènes de corrélations actives;

2) aux différences d'aptitudes à la croissance des diverses pointes de racines, ces aptitudes pouvant être déterminées par le complexe de corrélation dans lequel elles naissent;

3) à des variations de niveau d'un pool hormo-trophique utilisable par les racines.

\section{Introduction}

Le système racinaire de semis de chêne présente un épais pivot vertical, à croissance durable, sur lequel naissent des racines secondaires, fines et à croissance axiale géné-

R. Tavakol : actuellement Université de Hamadan, Iran. 
ralement limitée. C'est un type de système racinaire comportant des pointes de dimensions et à destins bien différenciés, que nous appellerons «système racinaire bien hiérarchisé » (fig. 1).

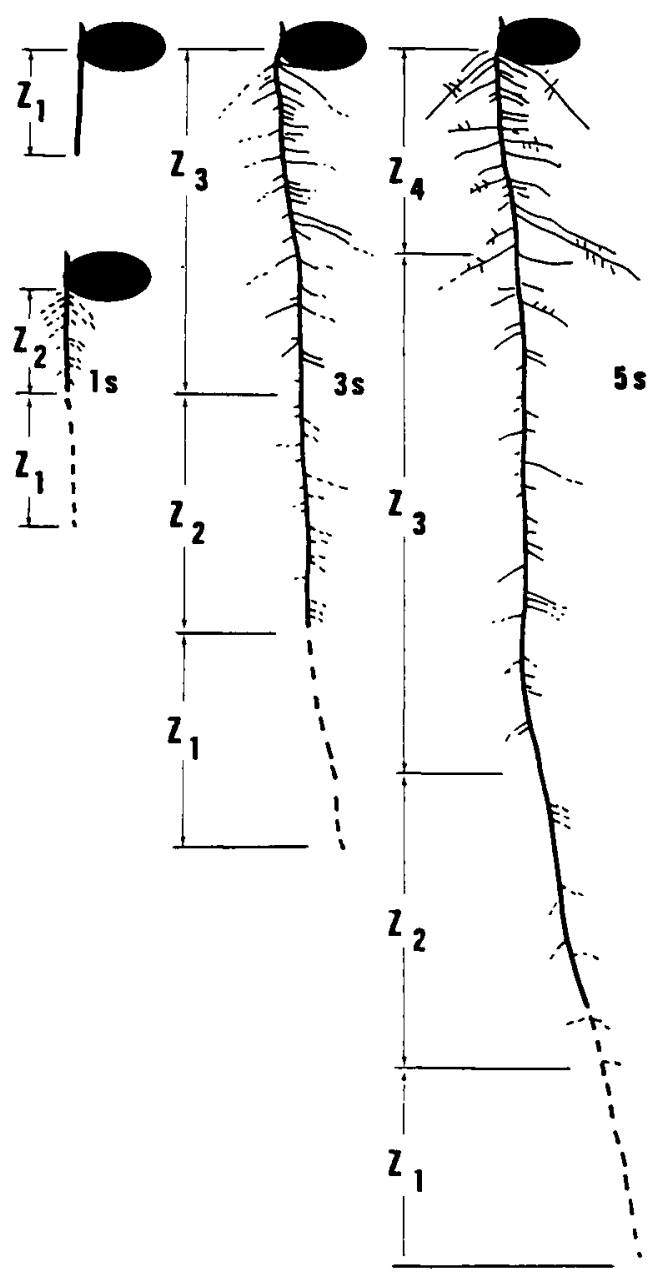

$4 \mathrm{~cm}$

Morphogénèse du système racinaire d'un semis de chêne pédonculé placé en minirhizotron après 1,3 et 5 semaines.

On notera quatre zones $(z)$ à partir de la $3^{e}$ semaine $: z_{1}$ : ancune racine latérale apparente; $z_{2}:$ toutes les racines latérales $s^{\prime} y$ allongent $; z_{*}:$ certaines racines latérales ont cessé de s'allonger: et $z_{1}$ : l'allongement de toutes les racines latérales est terminé ; - . - : allongement depuis le dernier relevé (en général 7 jours).

Morphogenesis of a root system of an oak seedling as it can be seen on the transparent side of a minirhizotron 1, 3 and 5 weeks after germination.

Four zones may be distinguished after 3 weeks : $z_{1}:$ no visible lateral roots; $z_{2}:$ all lateral roots extend. $z_{s}:$ some lateral roots no longer elongate, and $z_{k}:$ all lateral roots have stopped their elongation; ..... : the dotted line indicates growth during the previous week. 
A l'opposé, il existe des systèmes racinaires peu hiérarchisés dont les racines secondaires présentant des caractéristiques analogues à celle de la racine principale sont capables de remplacer facilement cette dernière. Tel est le cas du système racinaire des Cedrus atlantica que nous étudions par ailleurs (RiEDACKER, 1976).

Peu d'études ont cependant été effectuées pour comprendre le déterminisne d'arrêt de croissance des racines secondaires des systèmes racinaires des végétaux ligneux bien hiérarchisés, à part celles de Dyanat \& Neville (1970 et 1972) sur le cacaoyer et celles de Champagnat M. et al., 1974, de Lamond, 1979, Riedacker \& Poda, 1977, et Riedacker et al., 1982 sur les semis de chêne.

Dans le présent travail, nous avons cherché à préciser les corrélations de croissance existant entre le pivot (c'est-à-dire la racine primaire) et les racines secondaires. Pour cela, nous avons effectué des blocages d'une partie plus ou moins importante de l'exirémité distale de pivots de différentes longueurs.

\section{Matériel et méthodes}

Cette étude a été réaliséc avec des semis de chêne pédonculé (Quercus robur L.) issus de la germination de glands récoltés dans diverses régions de France (Creuse, 1976 ; HauteVienne, 1978 ; Hautes-Pyrénées, 1976 et 1977 ; Puy-de-Dôme, 1976 ct 1978). Ces glands ont été conservés jusqu'à utilisation, enrobés de rhodiazan, dans de la tourbe sèche, soit à $-1{ }^{\circ} \mathrm{C}$, soit à $4^{\circ} \mathrm{C}$. Leur mise en germination a été précédée de l'enlèvement des enveloppes séminales (péricarpe et téguments) et d'un lavage des embryons à l'eau courante pendant $48 \mathrm{~h}$. Ce traitement des semences réduit à quelques jours le délai de germination sans modifier la croissance et le développement ultérieur des semis (LAMOND, 1979).

Le type d'observations et de mesures qu'il est possible de réaliser sur des systèmes racinaires dépend du dispositif d'élevage des plants. Deux dispositifs d'élevage ont été utilisés : le minirhizotron (RIEDACKER, 1974) et le caisson à aérosol nutritif (LAMOND \& Mousain, 1979). Le minirhizotron a été employé pour préciser en particulier les directions de croissance, ainsi que les réactions géotropiques de chacune des racines visibles. Le caisson à aćrosol a été utilisé pour observer la dynamique de croissance de lat totalité du système racinaire et évaluer avec précision le nombre, la dimension, ainsi que le poids de ses différents composants.

Dans le cas des cultures effectuées sur minirhizotron, les embryons ont été posés à plat sur la tourbe fertilisée (DeLRan et al., 1975), immédiatement après leur lavage à l'eau courante. Dans le cas des cultures sur caisson à aérosol, ils ont été élevés sur vermiculite jusqu’à ce que leur pivot atteigne une longueur de 10 à $12 \mathrm{~cm}$.

L'élevage des semis en minirhizotrons a été réalisé au laboratoire $(5000$ Lux, 16 h d'éclairement, $18 \mathrm{~h}$ à $\left.24^{\circ} \mathrm{C}\right)$, tandis que celui sur aérosol a été réalisé en chambre climatisée (5000 Lux, $16 \mathrm{~h}$ d'éclairement, $25^{\circ} \mathrm{C}, 70 \pm 10 \%$ d'humidité relative).

Les traitements suivants ont été effectués chacun sur 10 individus élevés sur caisson et sur 6 individus élevés sur minirhizotron (fig. 2) :

- Plâtrage de l'extrémité de pivots sur une longueur de $1 \mathrm{~cm}$ pour bloquer leur croissance en longueur. Ce traitement a été effectué sur des pivots mesurant $6 \mathrm{~cm}$ (1 ou $\left.\mathbf{P}_{6}\right)$,

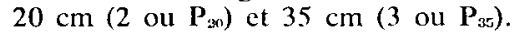

- Plâtrage de l'extrémité distale de pivots de $35 \mathrm{~cm}$ de long. sur des longueurs variables. Ce traitement bloque non seulement la croissance de la racine principale, mais encore celle de ses ramifications. Les portions suivantes du système racinaire ont été plâtrées :

- extrémité distale du pivot sur laquelle aucune ramification n'est encore visible (4 ou $\mathrm{P}_{w z n}$ ); 


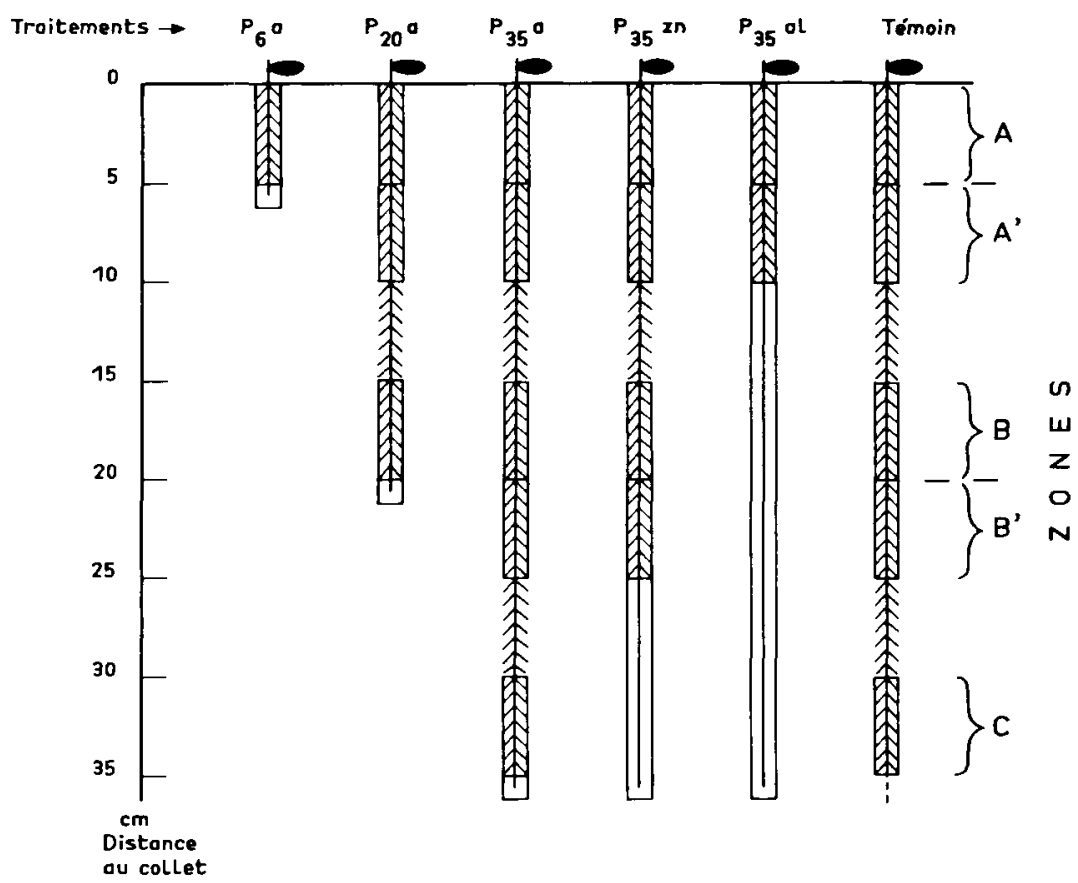

FIG. 2

Représentation schématique des différents traitements.

La zone du pivot enrobée de plâtre est représentée par un rectangle blanc. Les caractéristiques des racines latérales ont été mesurées dans les zones $A A^{\prime}, B B^{\prime}$ et $C$.

(1) $P_{6} a$, (2) $P_{s a} a$, (3) $P_{s 5} a$ : Pivot de 6,20 et $35 \mathrm{~cm}$ de longueur dont l'apex a été plâtré sur $1 \mathrm{~cm}$.

(4) $P_{s s} z n$ : Pivot de $35 \mathrm{~cm}$ de longueur dont toute l'extrémité ne portant pas de racines secondaires apparentes a été plâtrée.

(5) $P_{s i}$ al : Pivot de $35 \mathrm{~cm}$ de longueur dont toutes les racines secondaires qui étaient en train de s'allonger ont été plâtrées.

(6) $T:$ Témoin.

Schematic representation of the different treatments.

The white rectangle shows the plastered part of the taproot. Lateral root caracteristics have been measured in the zones $A A^{\prime}, B B^{\prime}$ and $C$.

(1) $P_{6} a$, (2) $P_{20} a$, (3) $P_{s s} a: 6-20$ and $35 \mathrm{~cm}$ long taproots with $1 \mathrm{~cm}$ long plastered tips.

(4) $P_{s s z n}: 35 \mathrm{~cm}$ long taproot of which the part of the tip having no visible lateral roots has been plastered.

(5) $P_{s s}$ al : $35 \mathrm{~cm}$ long taproot of which the tip and the part having elongating lateral roots has been plastered.

(6) $T$ : control not plastered. 
Nombre de

rocines secondaires

par plant
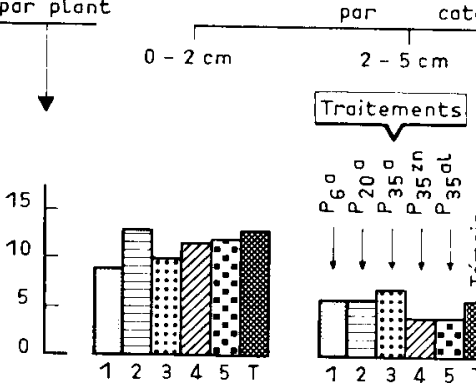

0 o 5 a

$a^{\infty} a^{\infty} a^{m} a^{m}$

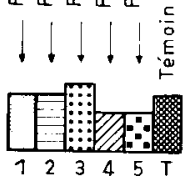

N I VEAUX

A
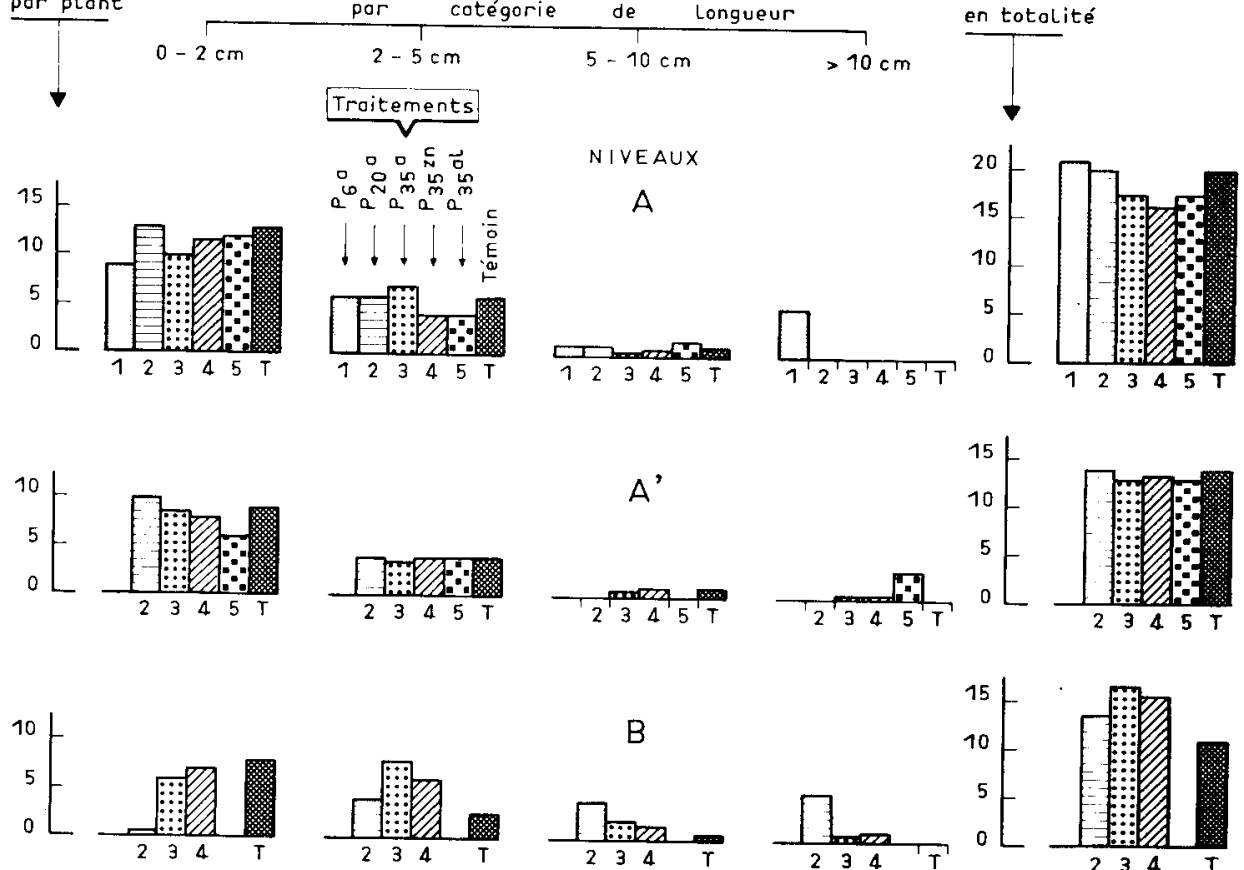

$\mathrm{B}$
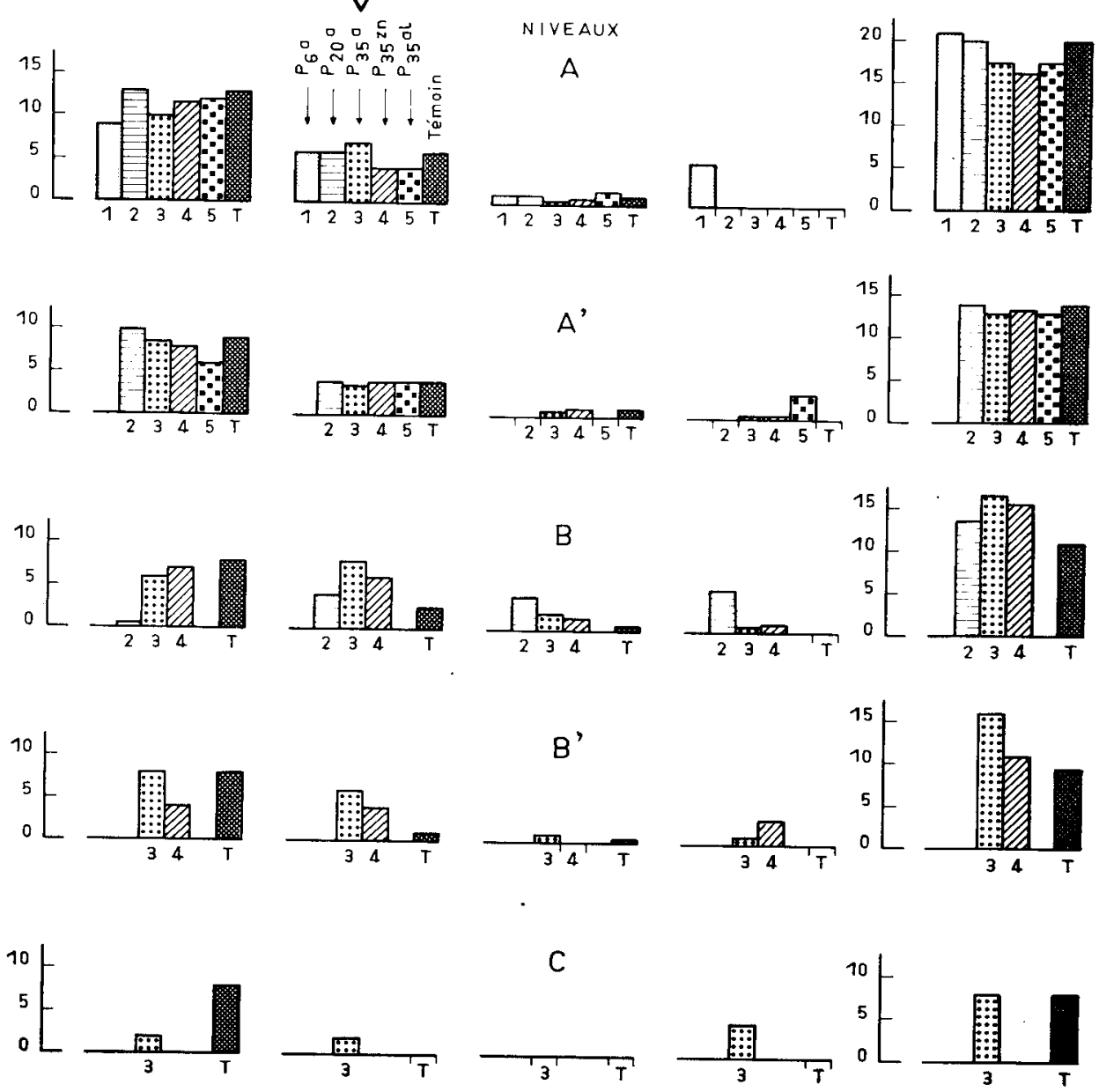

Fig. 3

Influence des différents traitements sur le nombre et la longueur des racines secondaires visibles sur minirhizotrons dans les zones $A, A^{\prime}, B, B^{\prime}$ et $C^{\prime} 2$ mois après le blocage des pivots. $A$ u centre, le nombre de racines secondaires visibles par catégorie de longueur, et à droite, la totalité des racines secondaires. Voir fig. 1 pour les traitements.

Influence of the different treatments on the number and the length of lateral roots visible on minirhizotrons in zone $A, A^{\prime}, B, B^{\prime}$ and $C^{\prime} 2$ months after blocking of the tip. See treatments on fig. 1 . 
- extrémité distale du système racinaire sur une longueur de $25 \mathrm{~cm}$, c'est-à-dire toute la zone dans laquelle les racines latérales présentent un allongement notable (5 ou $\left.P_{35} a l\right)$.

- Plant témoin dont la croissance du pivot n'a pas été gênée (6 ou T).

Pour éviter de traumatiser les racines latérales pendant le plâtrage du pivot, les parties non traitées ont été recouvertes d'un papier filtre humide lorsque les plants étaient élevés sur minirhizotrons, soit maintenues dans l'aérosol.

Pour étudier les réponses morphogénétiques consécutives à ces traitements, nous avons :

- dessiné chaque semaine les allongements (parties en pointillé sur les figures) des racines visibles sur une feuille de polyéthylène appliquée sur la face transparente du minirhizotron ;

- mesuré la longucur des racines latérales des zones $\mathrm{AA}^{\prime}, \mathrm{BB}^{\prime}$ et $\mathbf{C}$ (fig. 2) visibles sur minirhizotron après 3 mois;

- révélé la nature du géotropisme des racines latérales en faisant subir aux minirhizotrons une rotation de $90^{\circ}$ dans le plan vertical;

- mesuré 3 semaines après les opérations, le nombre, le poids sec et la longueur de la totalité des racines latérales des plants élevés sur aérosol et situées dans les 3 zones indiquées sur les figures 10 et 12.

\subsection{Morphogenèse du système racinaire des plants témoins}

Le système racinaire des semis témoins se compose d'une racine primaire verticale, de racines secondaires obliques ou horizontales et de nombreuses racines tertiaires plus ou moins perpendiculaires à leur axe support (fig. 1).

La racine primaire, ou pivot, est directement issue de la croissance de la radicule. Elle est orthogéotrope (RIEDACKER et al., 1982).

Le pivot s'allonge en général sans arrêt (fig. 4, courbe A) à une vitesse à peu près constante : $1,4 \pm 0,15 \mathrm{~cm} /$ jour à $18^{\circ}$ et $2,0 \mathrm{~cm} /$ jour à $25^{\circ} \mathrm{C}$. Sur quelques individus peu vigoureux, elle cesse parfois momentanément de s'allonger (fig. 4, courbe B). Ces arrêts de courtes durées (maximum 1 semaine) s'observent le plus souvent au cours de la quatrième ou cinquième semaine de culture, c'est-à-dire lorsque les réserves cotylédonnaires arrivent à épuisement (LAMOND, en préparation).

Le diamètre de l'apex du pivot, mesuré à $0,4 \mathrm{~cm}$ de la coiffe, varie entre 1,2 et $1,8 \mathrm{~mm}$. La portion basale, mesurée à $1 \mathrm{~cm}$ du collet, s'accroît régulièrement; son diamètre dépasse généralement $5 \mathrm{~mm}$ après 2 mois de culture.

Les racines secondaires, ou ramifications d'ordre 1, apparaissent normalement à une distance de 6 à $10 \mathrm{~cm}$ de l'apex du pivot. Elles sont semi-plagiogéotropes, mais jamais orthogéotropes (RIEDACKER et al., 1982).

La croissance en longueur de ces racines secondaires varie considérablement, mais demeure toujours limitée. Les plus grosses, en particulier celles insérées à une faible distance du collet, s'allongent pendant environ 3 semaines et peuvent atteindre une longueur voisine de $10 \mathrm{~cm}$. A l'opposé, les racines les plus fines cessent de s'allonger quelques jours seulement après leur émergence; leur longueur finale ne dépasse pas $1 \mathrm{~cm}$ (fig. 1).

Le diamètre de leur apex, mesuré à $2 \mathrm{~mm}$ de la coiffe, varie beaucoup d'une racine à l'autre $(0,09$ à $0,43 \mathrm{~mm}, 7$ à 10 jours après leur émergence) et diminue jusqu'à 
ce que la croissance axiale cesse. Seulement quelques-unes d'entre elles (10 à 15 p. 100) présentent un accroissement radial à leur base qui soit détectable. Mais, même ces dernières restent fines et leur diamètre mesuré à $5 \mathrm{~mm}$ du pivot ne dépasse généralement pas $0,63 \mathrm{~mm}$ après 2 mois de culture.

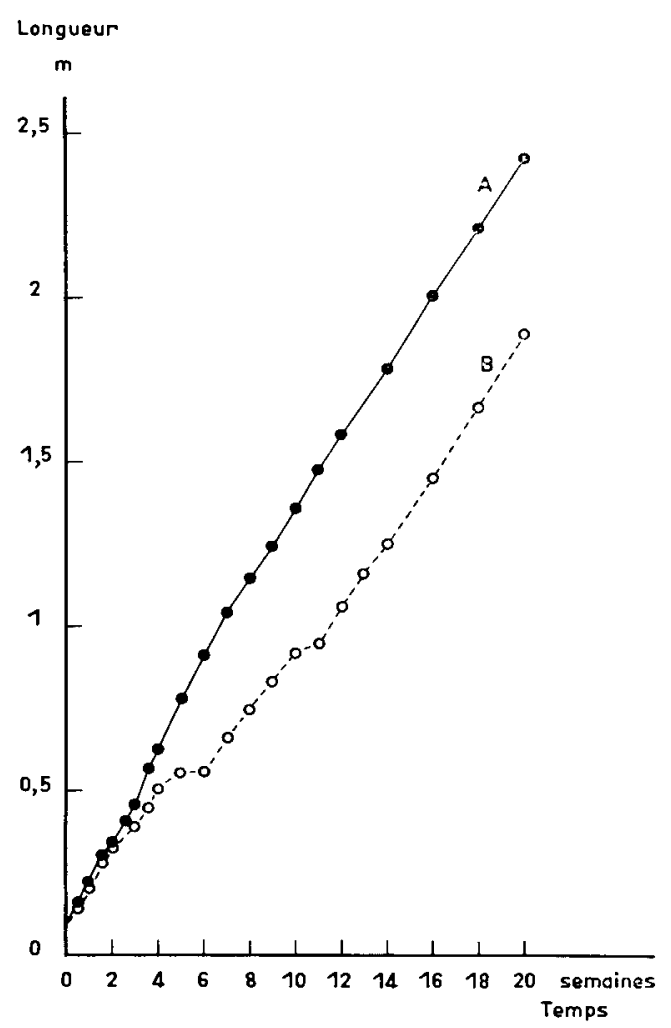

FIG. 4

Allongement de deux pivots élevés sur aérosols.

Le pivot $A$ ne présente pas de ralentissement de la vitesse d'allongement,

alors que le pivot $B$, moins vigoureux, en présente vers l'âge de 5 semaines et de 10 semaines.

Elongation of taproots in aeroponics.

Taproot $A$ has a constant elongation rate, whereas taproot $B$, less vigorous,

has an elongation rate declining at 5 and 10 weeks.

Lorsque les semis sont élevés sur minirhizotron, larrêt de croissance des racines secondaires est définitif. Par contre, lorsqu’ils sont cultivés sur caisson à aérosol nutritif et présentent une longueur satisfaisante, un bon nombre de racines latérales (généralement les plus grosses), recommencent à s'allonger vers la septième semaine de culture. La reprise de croissance s'effectue de manière synchrone sur un même plant, après gonflement des apex impliqués (fig. 5). La croissance axiale qui en résulte cesse assez rapidement et n'ajoute que quelques centimètres à leur longueur. 

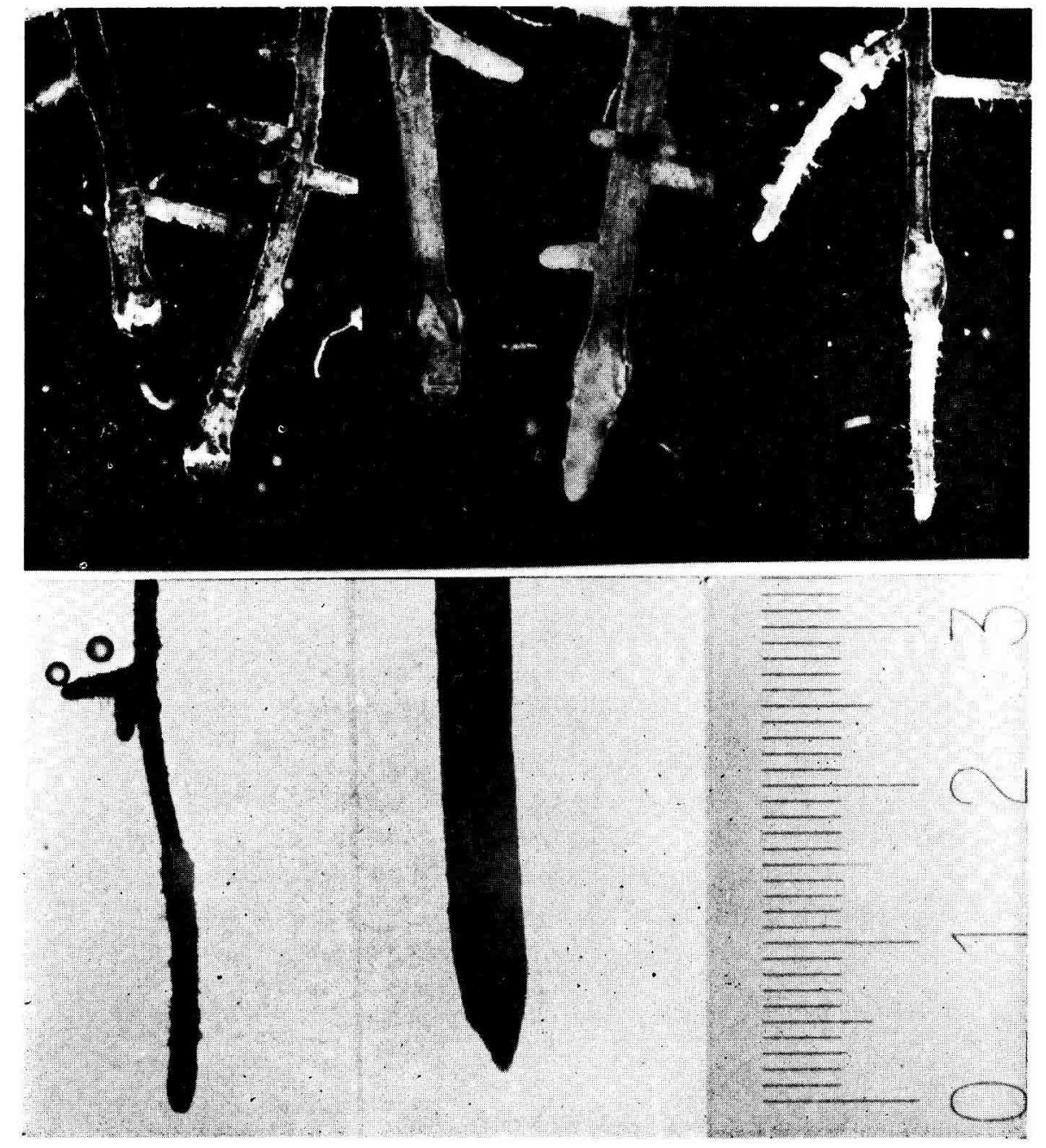

FIG. 5

Photographies montrant la reprise de croissance de racines secondaires.

- En haut à gauche : gonflement des pointes des racines.

- En haut au centre : racines au moment de la reprise d'allongement.

- En haut à droite : allongement.

- En bas : à la même échelle, à gauche, une racine secondaire ayant recommencé à s'allonger, et au centre une racine secondaire tardive apparue au moment de la reprise de croissance des racines secondaires.

Photography showing how lateral root growth resumes.

- Top left : thickening of the root tip.

- Top center : roots about to elongate.

- Top rigtht : extension growth.

- Bottom : on a same scale, a secundary root which resumed growth and in the center a late lateral root which appeared when the lateral roots started to grow again. 


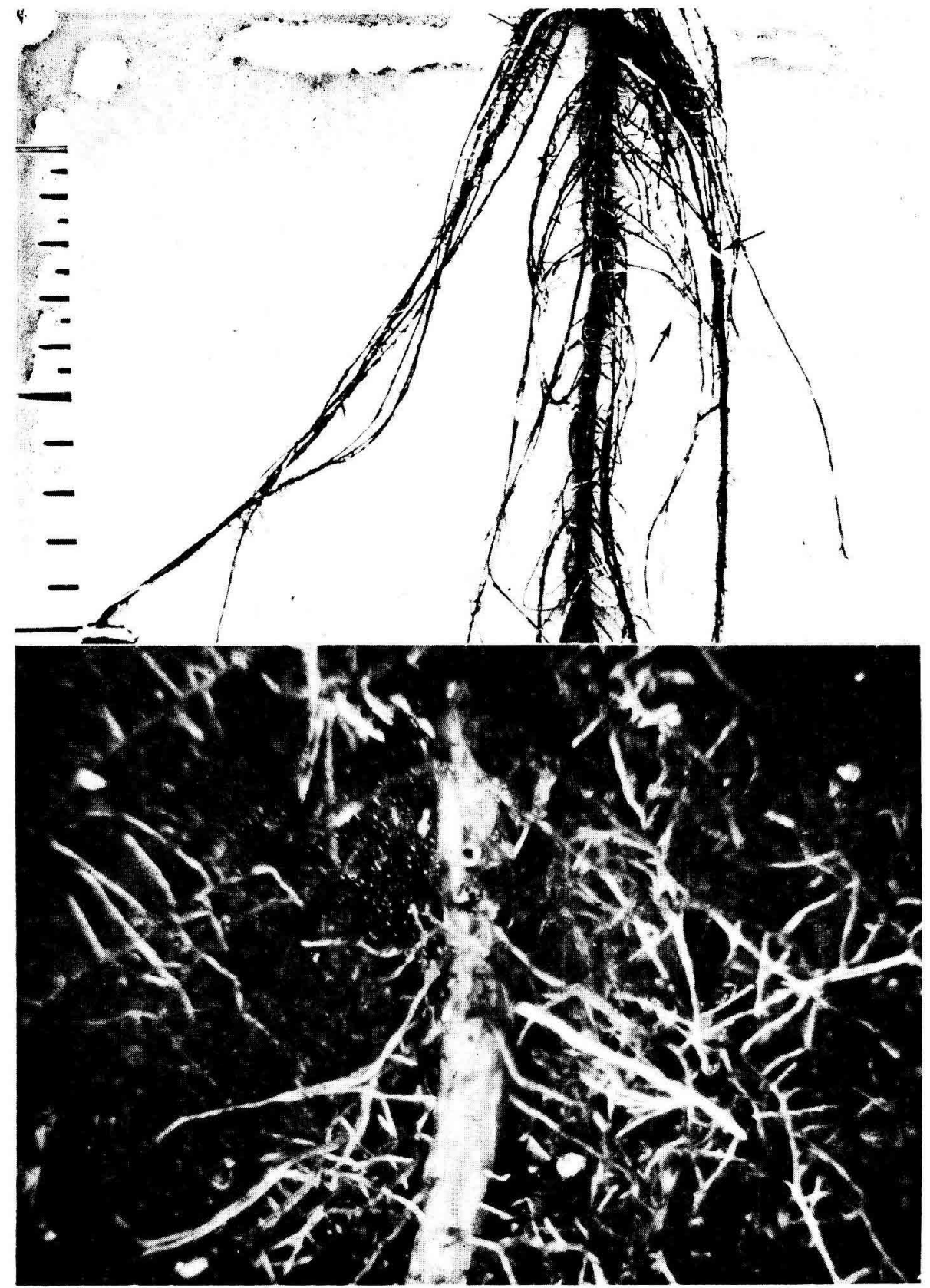

FIG. 6

Apparition de «racines secondaires tardives» blanches sur le pivot de jeunes chênes.

- En haut : sur un système racinaire de plant élevé sur aérosol nutritif, les racines secondaires apparues normalement en succession acropète au voisinage de l'apex du pivot sont foncées.

- En bas : racines secondaires tardives apparues sur tourbe à la suite d'un blocage du pivot. Noter également le développement important des racines tertiaires.

White «late lateral roots》 appearing on the taproot of young oak seedlings.

-- Top : on a root system grown in aeroponics the former lateral roots which appeared regularly behind the apex are brown.

- Bottom : late lateral roots which appeared on a blocked taproot in peat. Notice also the important development of tertiary roots. 
Cette reprise de croissance s'accompagne, dans la portion déjà ramifiée du pivot, de l'émergence de quelques racines latérales d'ordre 1 , tardives, dont le diamètre à la naissance est environ le double de celui des ramifications de même ordre initiées en succession acropète au voisinage de l'apex du pivot (fig. 6). Sur des plants âgés de 20 semaines, certaines d'entre elles atteignent une longueur voisine de $60 \mathrm{~cm}$ et un diamètre à la base de $1,5 \mathrm{~mm}$.

Les racines tertiaires apparaissent environ 25 jours après la germination. Elles croissent en succession acropète sur les racines secondaires les plus longues. Elles sont très fines, agéotropes, ne s'allongent que quelques jours, puis brunissent. Leur longueur dépasse alors rarement $5 \mathrm{~mm}$. Lorsque les plants sont élevés sur aérosol nutritif, leur pointe redevient temporairement blanche au moment de la reprise de croissance des racines secondaires.

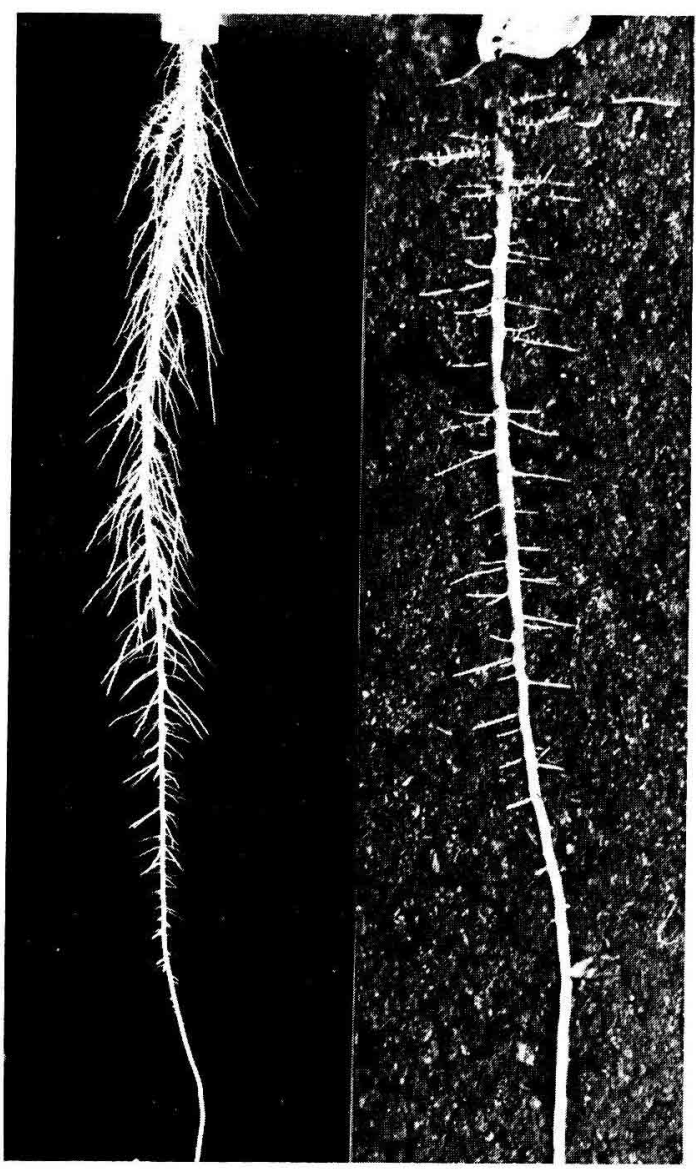

FIG. 7

Aspect d'un système racinaire de semis de chêne élevé sur aérosol à gauche et sur tourbe en minirhizotron à droite.

Aspect of root systems grown in aeroponics (left) and in peat in a minirhizotron (rigth). 
En résumé, l'appareil souterrain des semis de chêne pédonculé se présente comme un système bien hiérarchisé, dans lequel les différents ordres de croissance se distinguent nettement non seulement par leur taille, mais encore par la durée de leur période de croissance. Et, malgré les petites différences de croissance observées, la morphologie des systèmes racinaires cultivés sur tourbe (fig. 7 a) diffère très peu de celle obtenue sur aérosol (fig. 7 b).

\subsection{Influence du blocage de l'apex des pivots}

\subsection{Blocage d'un pivot de $6 \mathrm{~cm}$ de longueur}

Ce traitement ne modifie ni l'ordre d'apparition, ni le nombre des racines secondaires (fig. 3 et 8 ). Il influence peu ou pas la durée de croissance de la majorité de ces racines. Seules quelques-unes, situées au voisinage du point de blocage, sont stimulées. Deux à trois d'entre elles acquièrent une croissance indéfinie (du moins tant que l'extrémité apicale du pivot reste bloquée) deviennent orthogéotropes et ont une croissance en épaisseur importante, quoique inférieure à celle d'un pivot. Nous les appellerons des racines secondaires transformées. Après deux mois de culture, leur diamètre à $1 \mathrm{~cm}$ du pivot, est égal à la moitié de celui de ce dernier.

Des observations sommaires montrent :

- que leurs formations secondaires ligneuses sont alors nettement plus développées que celles des racines secondaires non stimulées;

- que leur gros vaisseaux, inexistants dans les racines secondaires non stimulées, sont cependant moins nombreux et de taille inférieure à ceux des pivots;

- que le diamètre de leur moelle est également plus important que chez les témoins, mais reste inférieur à celui du pivot.

Les racines latérales tertiaires se développent par ailleurs davantage sur les racines stimulées que sur les témoins. Environ un mois après le blocage, nous avons observé, sur la paroi transparente des minirhizotrons, l'apparition de quelques racines secondaires tardives, dont les caractéristiques sont semblables à celles qui ont été décrites chez les témoins élevés sur aérosol nutritif. La croissance axiale de ces racines tardives est oblique ou horizontale. Elle cesse environ 4 semaines après leur émergence.

\subsection{Blocage d'un pivot de $20 \mathrm{~cm}$ de longueur}

Ce traitement (fig. 3 et 9) ne modifie de façon appréciable ni la croissance des racines latérales visibles au moment du blocage, ni le nombre, ni l'ordre, ni la vitesse d'apparition des racines secondaires qui se formeront par la suite dans la portion non encore ramifiée du pivot. Il stimule par contre très nettement la croissance de ces dernières : deux à trois d'entre elles, situées très près du point de blocage, acquièrent une croissance indéfinie et deviennent orthogéotropes. Les racines tertiaires, portées par les racines fortement stimulées, peuvent atteindre jusqu'à $6 \mathrm{~cm}$ et porter des racines quaternaires.

Un mois après le blocage, on observe aussi bien sur tourbe que sur aérosols l'émergence en divers endroits de racines latérales tardives ayant des caractéristiques analogues à celles décrites précédemment. 


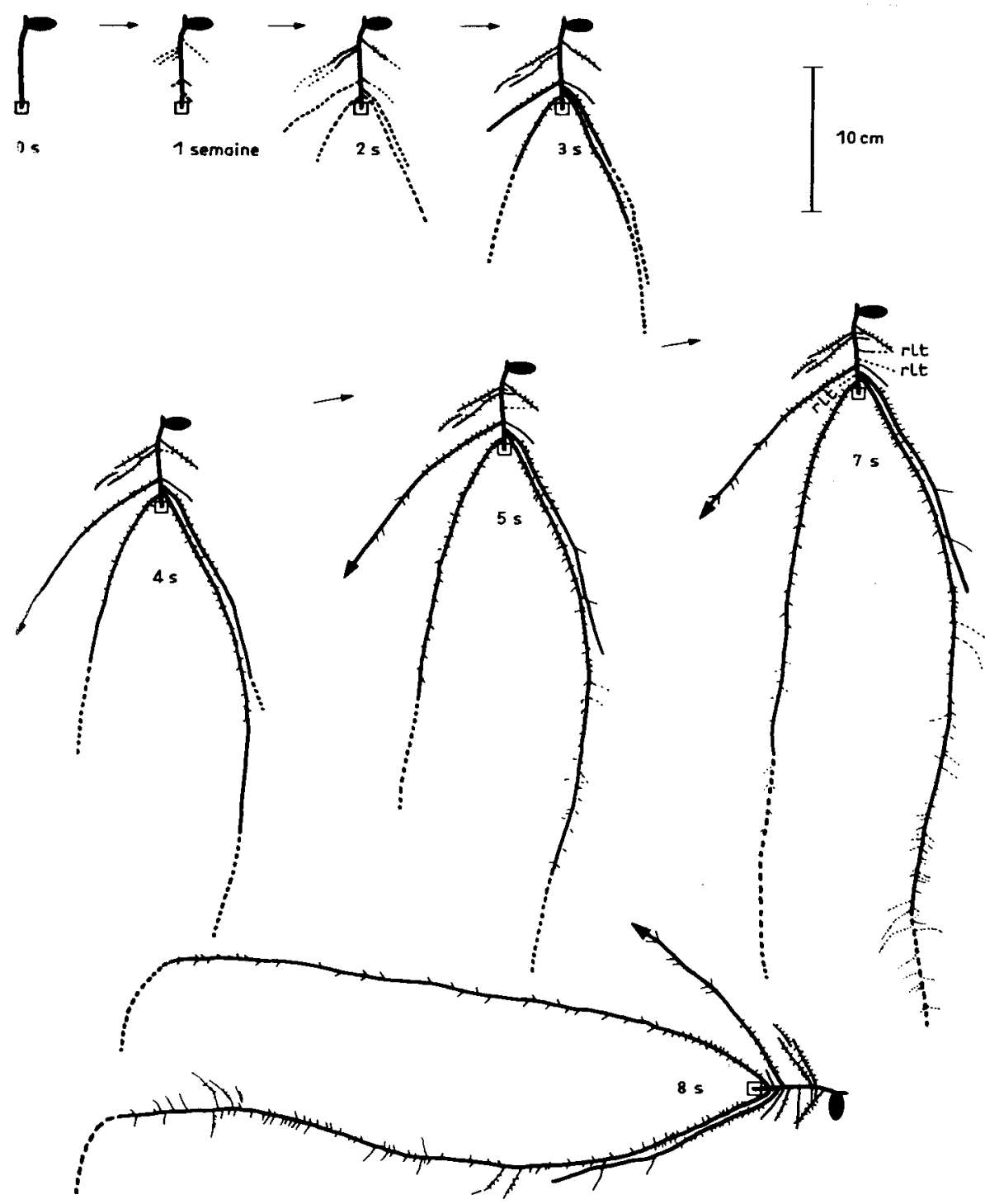

FIG. 8

Développement en minirhizotron du système racinaire d'un semis de chêne dont l'apex du pivot a été plâtré, alors qu'il avait une longueur de $6 \mathrm{~cm}$. Relevé hebdomadaire. On notera l'apparition des racines latérales tardives 7 semaines après le plâtrage.

le plan vertical.

- La flèche au bout des racines indique que leur pointe a disparu dans la tourbe. $r l t:$ racines latérales tardives 》.

Development in a minirhizotron of the root system of an oak seedling with an apex which was plastered when the taproot was $6 \mathrm{~cm}$ long. Weekly recording. Notice that late lateral roots appear 7 weeks after plastering.

- On the bottom, geotropic reactions of the roots after a $90^{\circ}$ rotation in a vertical plane.

- Arrows indicate that the tip has disappeared in the peat. rlt : "late lateral roots». 

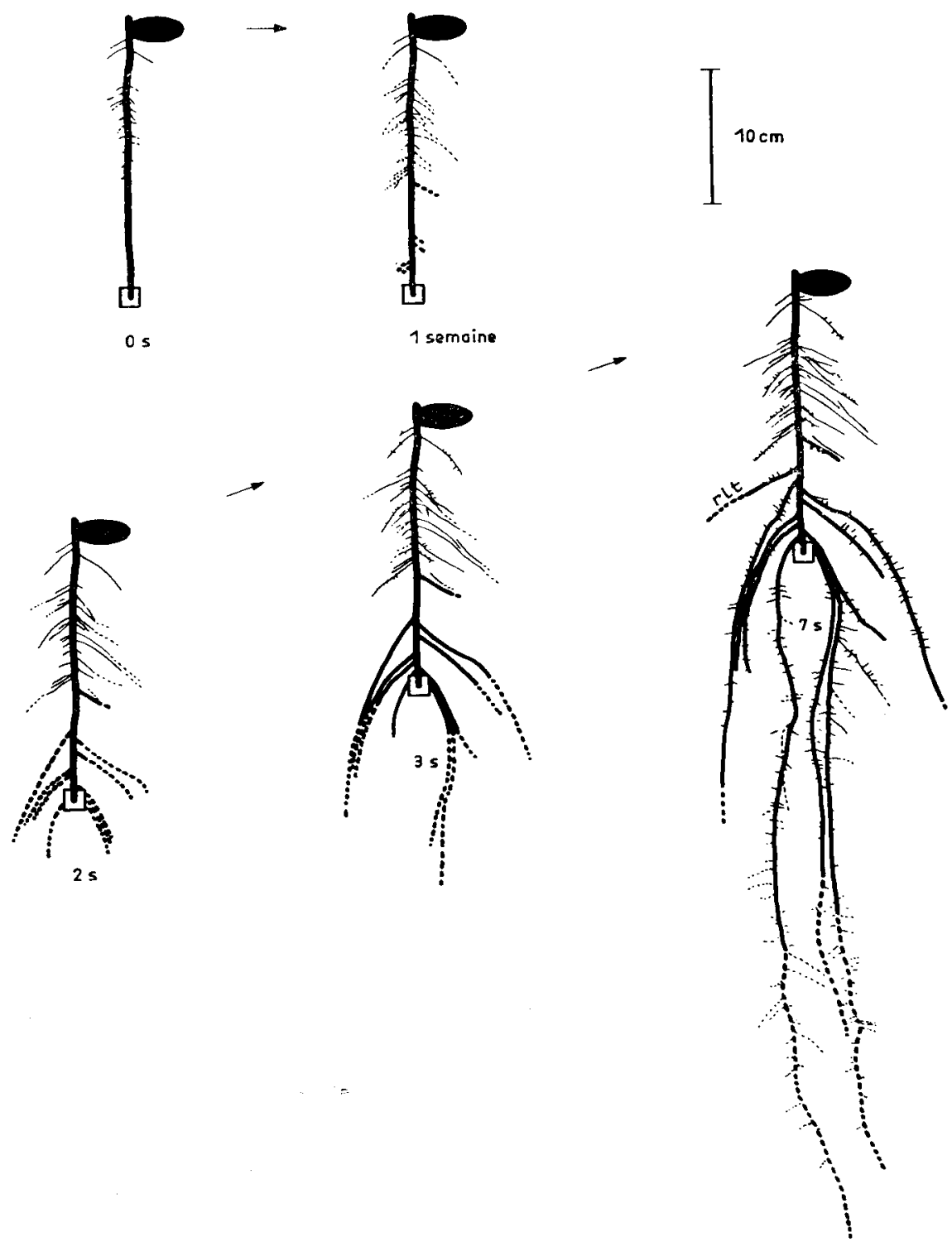

FIG. 9

Développement en minirhizotron du système racinaire d'un semis dont l'apex du pivot a été plâtré, alors qu'il atteignait une longueur de $20 \mathrm{~cm}$. Relevé hebdomadaire.

On notera l'apparition des racines latérales tardives 6 à 7 semaines après le plâtrage. Le temps en semaines est compté à partir du plâtrage.

Development in a minirhizotron of the root system of a seedling of which the apex was plastered when the taproot was $20 \mathrm{~cm}$ long. Weekly recording. Notice the appearance of late lateral roots 6 or 7 weeks after plastering. The time in weeks indicated begins with the plastering. 
Deux mois après le blocage, la fréquence des longueurs des racines secondaires situées dans la partie ramifiée du pivot au moment du blocage n'est pas différente de celle des témoins. Par contre, dans la partie du pivot non ramifiée au moment du blocage, on observe beaucoup moins de racines de 0 à $2 \mathrm{~cm}$ que chez les témoins, les manquantes se retrouvant en excès dans les classes de longueur supérieures.

Les mesures effectuées sur les racines latérales des semis cultivés sur caisson à aérosol nutritif, 21 jours aprè le blocage de pivot de même longueur, conduisent à des conclusions analogues. Elles permettent en outre de préciser que la stimulation moyenne de la croissance en matière sèche des racines de la portion située entre 0 et $3 \mathrm{~cm}$ du blocage est voisine de 630 p. 100 (fig. 10). Cet accroissement pondéral est dû non seulement à l'allongement, mais encore à un accroissement radial plus important (fig. 11). L'anatomie des racines les plus fortement stimulées est modifiée comme dans le traitement précédent.
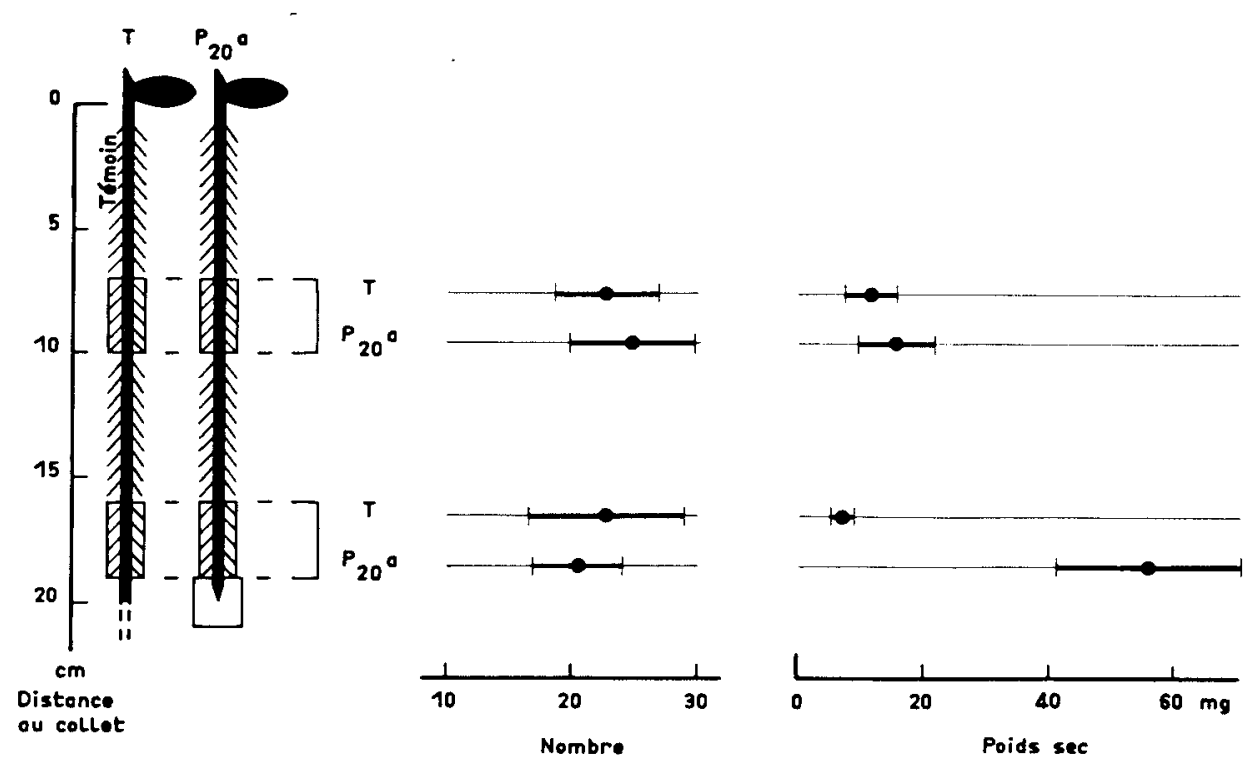

Rocines secondoires

FIG. 10

Influence du blocage d'un pivot de $20 \mathrm{~cm}$ de long d'un plant élevé sur aérosol nutritif, sur le développement des racines secondaires (en nombre et en poids sec).

Influence of blocking of $20 \mathrm{~cm}$ long taproots grown in aeroponics on the development of secundary roots (number and dry weigth).

FiG. 11

Influence du blocage de l'apex des pivots de $20 \mathrm{~cm}$ de long de plants élevés sur aérosol nutritif sur le développement des racines secondaires.

Les caractéristiques des plants témoins sont représentées par des rectangles blancs, et celles des plans dont l'apex a été plâtré par des rectangles grisés.

- Histogramme du haut : croissance en longueur des racines secondaires.

- Histogramme du milieu : diamètre des racines secondaires à la base du pivot.

- Histogramme du bas : diamètre des apex des racines secondaires. 

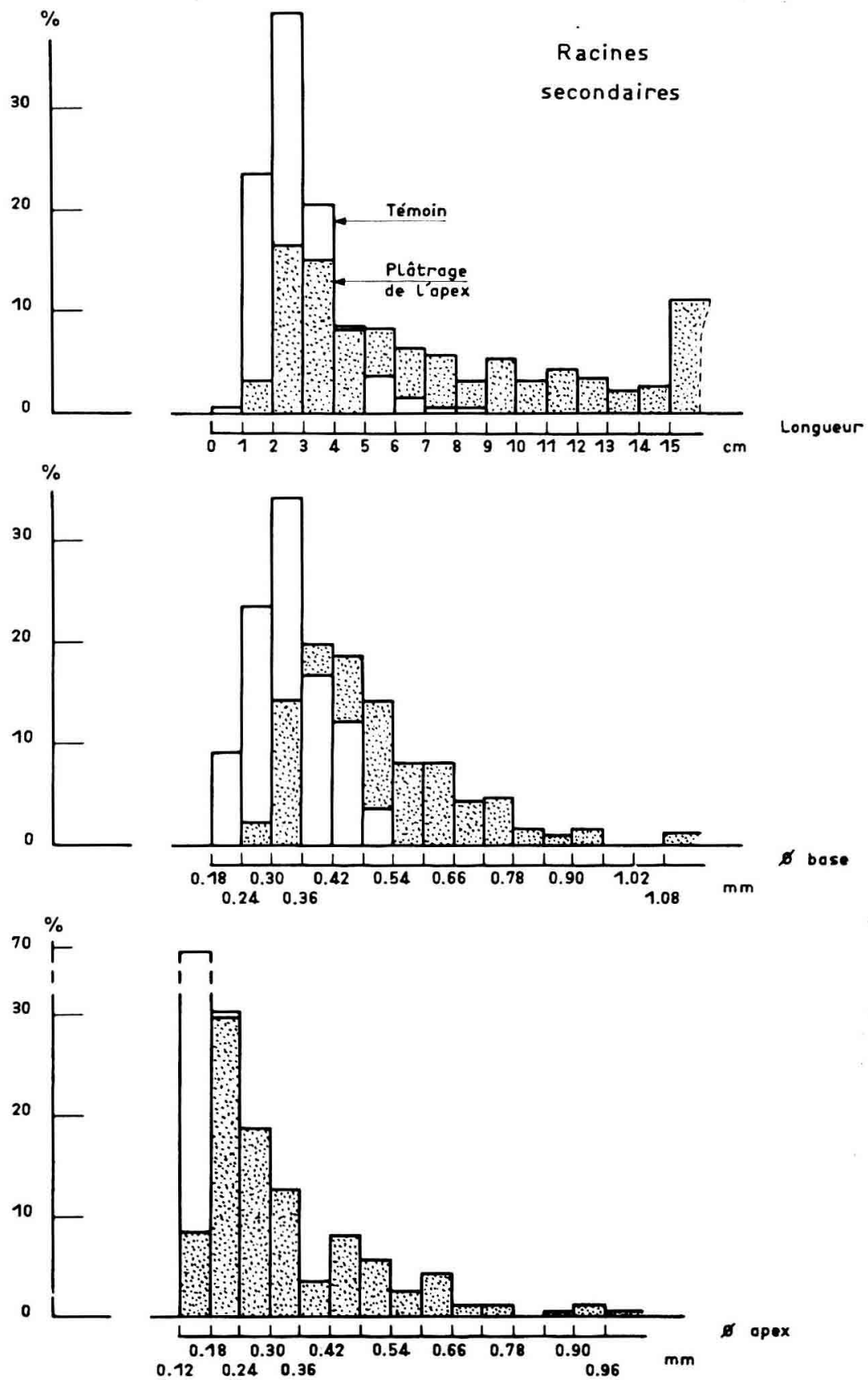

Effect of blocking of the apex of $20 \mathrm{~cm}$ long taproots grown in aeroponics, on the development of lateral roots.

White rectangles are for control root systems and grey rectangles for root systems with a plastered apex.

- Top : effect on extension growth of secundary roots.

- Middle : effect on the basal root diameter of secundary roots.

- Bottom : effect on the apex diameter of secundary roots. 


\title{
2.23. Blocage d'un pivot de $35 \mathrm{~cm}$ de longueur
}

Lorsque le pivot atteint $35 \mathrm{~cm}$ de longueur, un certain nombre de racines secondaires situées au voisinage du collet ont cessé de s'allonger. Le blocage de l'extrémité distale du pivot n'entraîne pas la reprise de croissance de ces dernières. Il ne modifie pas non plus de façon appréciable la croissance des autres racines visibles au moment du blocage. Et, il stimule la croissance des racines qui se forment dans la zone non ramifiée du pivot au moment du blocage, de manière tout à fait analogue à celle du traitement précédent (fig. 3 et 12 et tabl. 1). Toutefois, ce blocage ne provoque pas l'apparition de racines latérales tardives au cours des deux premiers mois de culture.

\section{Influence du blocage d'une portion plus importante du système racinaire de semis dont le pivot atteint $35 \mathrm{~cm}$ de longueur}

\author{
3.1. Blocage de toute l'extrémité distale du pivot \\ sur laquelle aucune ramification n'est encore visible $\left(\mathrm{P}_{3, ;}, \mathrm{zn}\right)$
}

Ce traitement n'entraîne pas la reprise de croissance des racines secondaires qui avaient cessé de s'allonger. Les racines secondaires encore en croissance, situées à plus de $5 \mathrm{~cm}$ de la partie bloquée, sont légèrement stimulées. Celles situées immédiatement au-dessus de la partie bloquée sont stimulées plus longuement, acquièrent une croissance plus durable. Quelques-unes d'entre elles, c'est-à-dire celles qui étaient au départ les plus grosses, s'allongent jusqu'en fin d'expérience et deviennent orthogéotropes (fig. 12 et tabl. 1).

Une dizaine de jours après le blocage, il apparaît généralement une ou deux racines tardives au voisinage immédiat de la partie bloquée. Leur apex est dès la naissance plus gros que celui des autres racines secondaires qui se sont transformées. Elles présentent également une croissance radiale plus forte que ces dernières.

\subsection{Blocage de toute la zone du système racinaire dans laquelle les racines latérales présentent un allongement notable $\left(\mathrm{P}_{3 \pi} \mathrm{al}\right)$}

A la suite de ce traitement, aucun allongement de racines n'est détectable pendant une dizaine de jours. Puis, bon nombre de racines secondaires recommencent à s'allonger pendant quelques semaines (fig. 13). Parmi ces dernières, quelques-unes continuent à s'allonger jusqu'à la fin de l'expérience. Le diamètre de leur portion, formée après la reprise de croissance, est plus gros que celui de leur partie formée antérieurement (fig. 5), mais reste néanmoins plus faible que celui des racines secondaires apparues après blocage dans les traitements $\left(\mathrm{P}_{3: 5} \mathrm{a}\right.$ et $\left.\mathrm{P}_{\underline{20}, \mathrm{a}} \mathrm{a}\right)$.

\section{FiG. 12}

Action sur le nombre et le poids sec de racines secondaires dans différentes zones du pivot de plants élevés sur caisson à aérosol nutritif et ayant subi différents traitements : T, $P_{s i} a, P_{3 ;} z$ en et $P_{s 5} a l$.

Effect of various treatments on the number and dry weigth of secundary roots grown in aeroponics: $T, P_{s s} a, P_{s i} z n$ and $P_{s 5} a l$. 


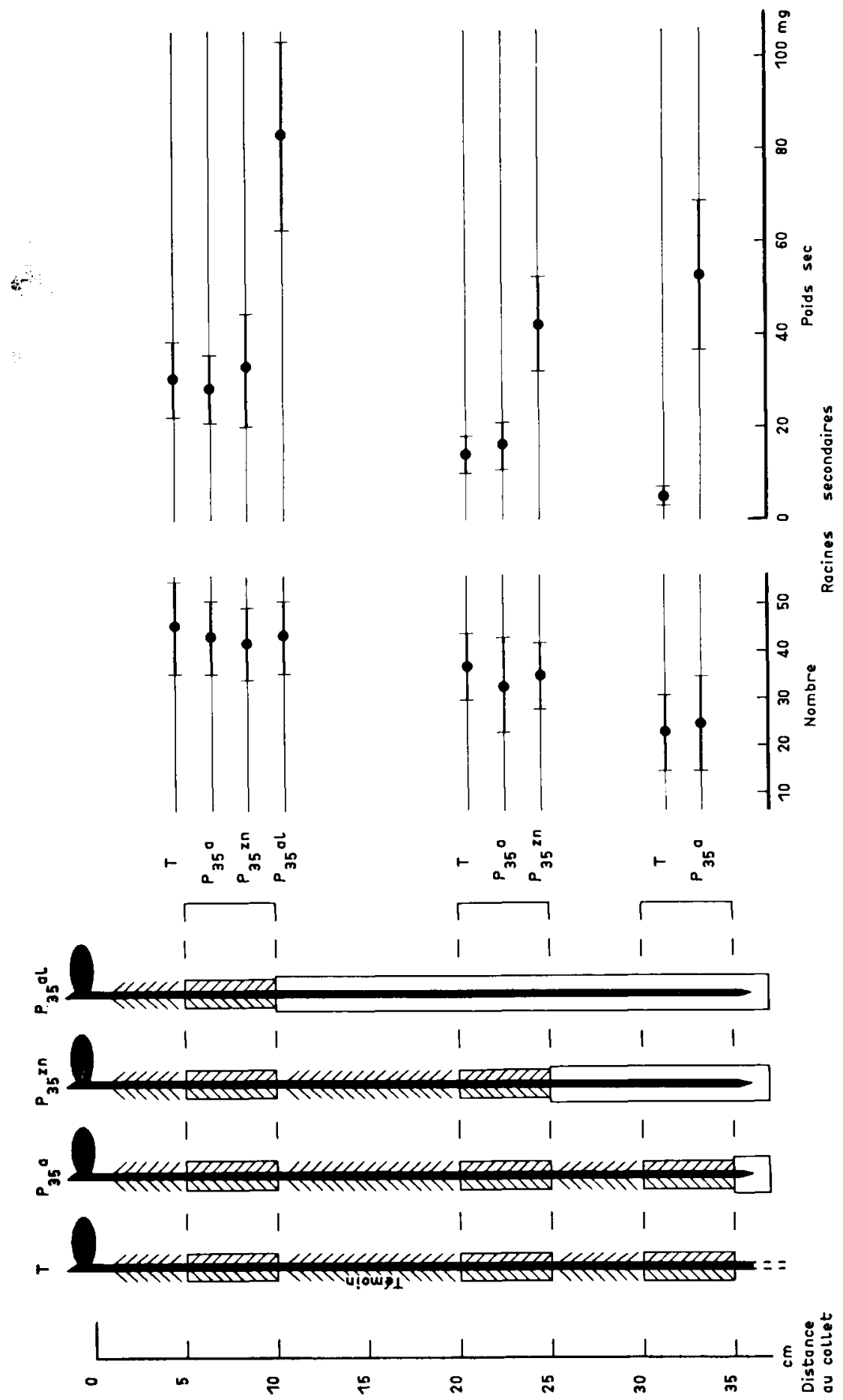




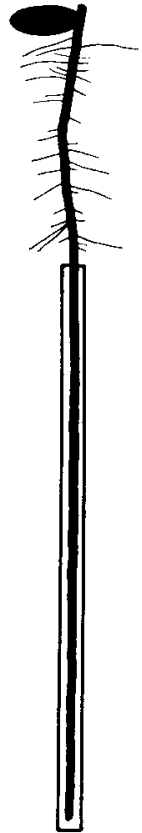

0 s

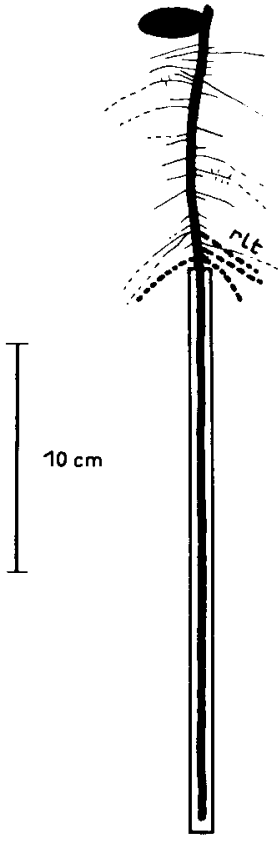

3,5 semaines

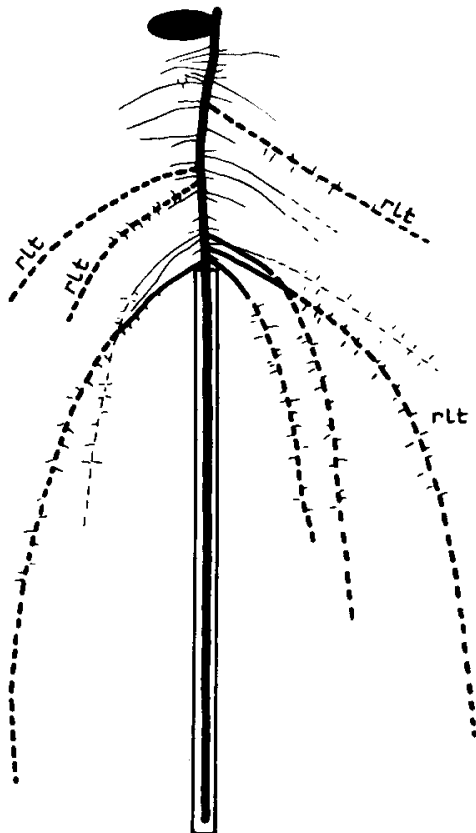

$7 \mathrm{~s}$

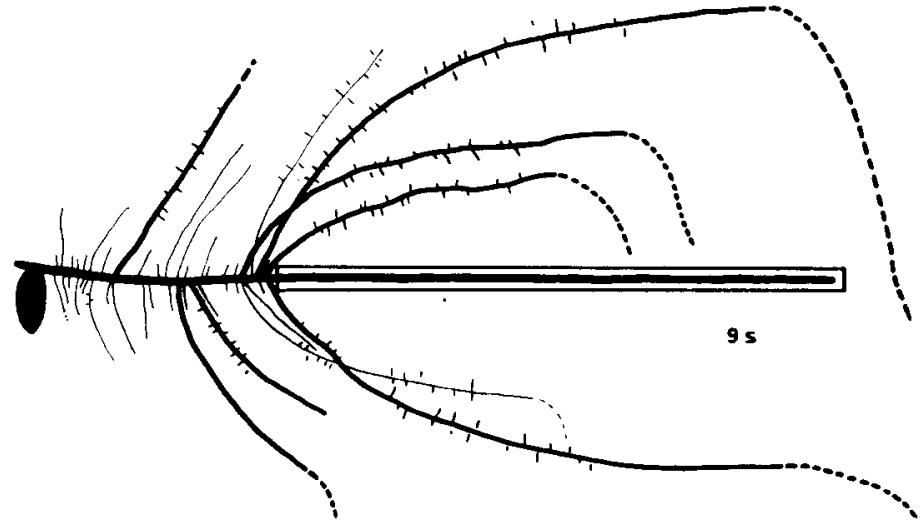

FIG. 13

Influence du plâtrage de toute la zone portant des racines secondaires en croissance sur le développement des racines secondaires d'un plant élevé en minirhizotron.

Influence of plastering the zone of the taproot with elongating secundary roots on the development of lateral roots in a minirhizotron. 


\section{TABLEAU 1}

Influence d'un blocage de l'extrémité distale du système racinaire sur la croissance en longueur des racines secondaires.

Semis cultivés sur minirhizotron $(A)$ ou sur caisson à aérosol nutritif $(B)$ et dont le pivot mesurait $35 \mathrm{~cm}$ de longueur au moment du traitement.

(Les fréquences sont exprimées en \%).

Influence of the blocking of the distal extremity of the root system on the apical growth of secondary roots.

Seedlings with a tap root length of $35 \mathrm{~cm}$ at the time of treatment were grown in a «minirhizotron 》 $(A)$ or in a box with nutrients supplied by aerosol (relative frequencies \%).

\section{A) Minirhizotrons}

\begin{tabular}{|c|c|c|c|c|c|}
\hline \multirow{2}{*}{$\begin{array}{l}\text { Zone d'insertion } \\
\text { des racines } \\
\text { secondaires } \\
\text { (distance à partir } \\
\text { du collet) }\end{array}$} & \multirow{2}{*}{$\begin{array}{c}\text { Classes } \\
\text { de longueur } \\
\text { des racines } \\
\text { secondaires } \\
(\mathrm{cm})\end{array}$} & \multicolumn{4}{|c|}{$\begin{array}{c}\text { \% de racines secondaires dans chaque classe } \\
\text { suivant le traitement }\end{array}$} \\
\hline & & Témoin & $P_{35} a$ & $P_{35 n} n$ & $P_{35}$ al \\
\hline $5-10$ & $\begin{array}{r}0-2 \\
2-5 \\
5-10 \\
>10\end{array}$ & $\begin{array}{r}64,2 \\
28,6 \\
7,2 \\
-\end{array}$ & $\begin{array}{r}65,4 \\
26,9 \\
5,4 \\
2,3\end{array}$ & $\begin{array}{r}60,3 \\
30,0 \\
7,5 \\
2,2\end{array}$ & $\begin{array}{c}48,0 \\
32,0 \\
-20,0\end{array}$ \\
\hline $20-25$ & $\begin{array}{l}0-2 \\
2-5 \\
5-10 \\
>10\end{array}$ & $\begin{array}{r}84,4 \\
10,5 \\
6,5 \\
- \\
\end{array}$ & $\begin{array}{r}50,2 \\
37,4 \\
6,2 \\
6,2 \\
\end{array}$ & $\begin{array}{c}30,7 \\
30,7 \\
\overline{38,6}\end{array}$ & \\
\hline $30-35$ & $\begin{array}{l}0-2 \\
2-5 \\
5-10 \\
>10\end{array}$ & $\frac{100}{-}$ & $\begin{array}{l}57,2 \\
14,3 \\
- \\
28,5\end{array}$ & & \\
\hline
\end{tabular}

B) Caisson à aérosol nutritif

\begin{tabular}{|c|c|c|c|c|c|}
\hline \multirow{2}{*}{$\begin{array}{l}\text { Zone d'insertion } \\
\text { des racines } \\
\text { secondaires } \\
\text { (distance en cm } \\
\text { par rapport au collet) }\end{array}$} & \multirow{2}{*}{$\begin{array}{l}\text { Classes } \\
\text { de longueur } \\
\text { des racines } \\
\text { secondaires } \\
(\mathrm{cm})\end{array}$} & \multicolumn{4}{|c|}{$\begin{array}{c}\% \text { de racines secondaires dans chaque classe } \\
\text { suivant le traitement }\end{array}$} \\
\hline & & Témoin & $P_{35} a$ & $P_{35} Z n$ & $P_{35} \mathrm{al}$ \\
\hline $5-10$ & $\begin{array}{r}0-2 \\
2-5 \\
5-10 \\
10-15 \\
>15\end{array}$ & $\begin{array}{r}42,0 \\
54,7 \\
3,3 \\
- \\
- \\
\end{array}$ & $\begin{array}{r}43,0 \\
50,6 \\
5,6 \\
0,8 \\
-\end{array}$ & $\begin{array}{r}41,8 \\
43,6 \\
12,6 \\
2,0 \\
-\end{array}$ & $\begin{array}{r}19,5 \\
42,5 \\
28,3 \\
5,9 \\
3,8\end{array}$ \\
\hline $20-25$ & $\begin{array}{r}0-2 \\
2-5 \\
5-10 \\
10-15 \\
>15 \\
\end{array}$ & $\begin{array}{r}80,5 \\
16,3 \\
3,2 \\
- \\
- \\
\end{array}$ & $\begin{array}{r}70,9 \\
26,4 \\
2,7 \\
- \\
-\end{array}$ & $\begin{array}{r}42,9 \\
37,1 \\
7,2 \\
6,8 \\
6,0 \\
\end{array}$ & \\
\hline $30-35$ & $\begin{array}{r}0-2 \\
2-5 \\
5-10 \\
10-15 \\
>15\end{array}$ & $\begin{array}{l}86,2 \\
13,8 \\
- \\
- \\
-\end{array}$ & $\begin{array}{r}47,9 \\
27,2 \\
8,7 \\
6,8 \\
9,4\end{array}$ & & \\
\hline
\end{tabular}


Simultanément à cette reprise de croissance des racines secondaires, il apparaît des racines secondaires tardives. Le diamètre de leur apex est environ deux fois plus gros que celui des racines latérales après leur reprise de croissance. Leur croissance radiale est plus forte et elles sont obliques. Les plus distales acquièrent une croissance indéfinie et deviennent orthogéotropes, tandis que les plus proches du collet restent obliques.

Par la suite, il apparaît également diautres racines secondaires tardives en d'autres endroits de la partie non bloquée du pivot, mais leur croissnce est moins durable que celle des précédentes (tabl. 1).

\section{Discussion}

Les expériences qui viennent d’être décrites montrent qu'un blocage de l'allongement du pivot entraîne une stimulation importante de la croissance des racines secondaires les plus distales et les plus grosses dès leur naissance. Mais, leur vitesse d'allongement reste longtemps inférieure à celle des pivots placés dans les mêmes conditions thermiques. Leur croissance en épaisseur est stimulée, mais reste aussi longtemps inférieure à celle des pivots. Leur géotropisme est enfin modifié. De plagiogéotropes, elles deviennent orthogéotropes, mais leur orthogéotropisme est plus faible que celui des pivots. Le rayon de leurs courbures géotropiques est en effet plus grand que celui des pivots (Riedacker et al., 1982).

Quelles que soient les longueurs de plâtrage, ce sont toujours les racines libres les plus distales qui sont les plus stimulées. Et, parmi elles, ce sont les racines les plus grosses dès leur naissance qui auront la croissance en longueur la plus importante et la plus durable.

Notons enfin que la décapitation de la pointe du pivot $(0,5 \mathrm{~cm})$ avant les plâtrages (résultats non publiés) modifie peu le comportement des racines secondaires.

On peut donc penser qu'il existe d'une part des corrélations actives entre le pivot et les racines secondaires et d'autre part une certaine hiérarchie entre les racines secondaires, cette hiérarchie dépendant en grande partie de la taille des racines à leur naissance. En effet, lorsque l'allongement du pivot n'est ni bloqué, ni freiné, les racines secondaires restent toutes courtes et fines. Mais, il existe cependant une certaine variabilité au sein de cette population des racines : certaines sont plus longues et plus grosses que d'autres. Lorsque l'allongement du pivot est réduit ou annulé, les racines secondaires peuvent se développer davantage. Les différences entre les racines secondaires s'amplifient alors et ce sont les racines les plus grosses qui sont les plus stimulées. Ainsi, lorsqu'on ne laisse libre que la zone du pivot près du collet, où avait cessé tout allongement de racines, on obtient à la fois une stimulation des racines secondaires et l'émergence tardive de nouvelles racines secondaires dont l'apex est d'emblée beaucoup plus gros que celui des racines préexistantes. Et ce sont précisément ces racines secondaires «tardives》qui se développeront le plus.

On peut aussi se demander pourquoi il apparaît davantage de racines secondaires tardives dans certaines circonstances. Lorsque le pivot n'est pas bloqué, il n'apparaît en effet pas de racines latérales tardives sur les plants élevés en minirhizotrons. En revanche, lorsqu'il est plâtré depuis la pointe jusqu'à la zone où les racines secondaires ont cessé 
de s'allonger, il apparaît de nombreuses racines secondaires tardives. Lorsque la zone nue des pivots élevés en minirhizotrons est plâtrée, il en apparaît également quelquesunes. Cela suggère que ces racines secondaires tardives n’apparaîtraient que lorsque les autres racines sont incapables «d'utiliser» la totalité du pool nutritif et hormonal disponible pour le système racinaire. Le fait que des racines latérales tardives apparaissent même sur les pivots des plants témoins élevés en aérosol nutritif, donc sur des plants dont l'alimentation hydrominérale est sans doute constamment supérieure à ce qu'clle est dans les minirhizotrons, nous paraît être un argument en faveur de cette hypothèse.

D'ailleurs, lorsqu'on ne bloque que la pointe du pivot, il apparaît peu de racines secondaires tardives. Cela pourrait être dû au fait que les racines secondaires qui croissent après le blocage sont d'emblée transformées, c'est-à-dire ont un diamètre plus gros et une croissance indéfinic leur permettant d'utiliser la presque totalité du pool hormono-trophique.

Lorsqu'on plâtre le pivot et toutes les racines en croissance, on constate à la fois une stimulation des racines secondaires qui avaient cessé de s'allonger et une apparition de racines secondaires tardives. Ces deux types de racines diffèrent néanmoins par la taille de leur apex et par leur diamètre.

Bien que cette stimulation augmente fortement le diamètre des apex des racines secondaires préexistantes, celui-ci reste inférieur à celui des racines secondaires tardives. Elles arrêteront rapidement de s'allonger. Leur transformation est d'ailleurs peut-être d'autant moins susceptible de s'opérer rapidement qu'il existe déjà un tronçon de racine secondaire très fin dont la croissance radiale initiale était très faible. La supériorité initiale des racines secondaires tardives pourrait être due à la fois au fait qu'elles ont un diamètre d’apex plus grand et pas de tronçon fin préexistant.

La zone la plus stimulée qui s'étend sur cinq à dix centimètres est toujours située dans la partie la plus distale. A priori, on pourrait done penser quil existe une certaine polarité. Mais, cette polarité est-elle responsable du fait que ce sont les racines les plus distales qui se développent le plus, ou est-elle responsable seulement du fait que ce sont les racines les plus distales qui, croissant après un blocage, ont aussi les plus gros apex, ce qui les rendrait les plus compétitives? Les deux hypothèses qui ne sont pas contradictoires semblent pouvoir être retenues pour l'instant.

Sur le plan pratique, il apparaît que le blocage de la pointe du pivot permet d'obtenir un système racinaire ayant des racines charpentières distribuées différemment de celles d'un pivot décapité (fig. 14) et par conséquent susceptibles d'explorer un volume plus important de sol. Ce résultat pourrait être mis à profit pour modifier la morphogénèse du système racinaire pivotant pendant l'élevage, soit lorsqu'il est impossible de soulever les plants sur place, notamment lorsque le sol est caillouteux, ou lorsque les plants sont élevés en conteneurs. Le blocage du pivot pourrait avoir lieu à 5 ou $6 \mathrm{~cm}$ du gland. Cette opération permet également de stimuler le développement de racines tertiaires, donc d'améliorer le chevelu racinaire (fig. 6). Il n'est pas impossible que cela puisse être un facteur favorable à la mycorhization.

Le blocage, contrairement à la dessication de la pointe du pivot arrivant à l'air libre (RIEDACKer et al., 1982), permet enfin d'obtenir des racines secondaires orthogéotropes susceptibles de coloniser le sol en profondeur lorsque les conditions édaphiques le permettent. Il permettrait sans doute aussi d'obtenir un système racinaire mieux 
adapté à la colonisation des sols superficiels ou hydromorphes. Le blocage paraît également plus intéressant que la déviation qui ne permet d’obtenir qu'un pivot coudé et un développement limité des racines secondaires.
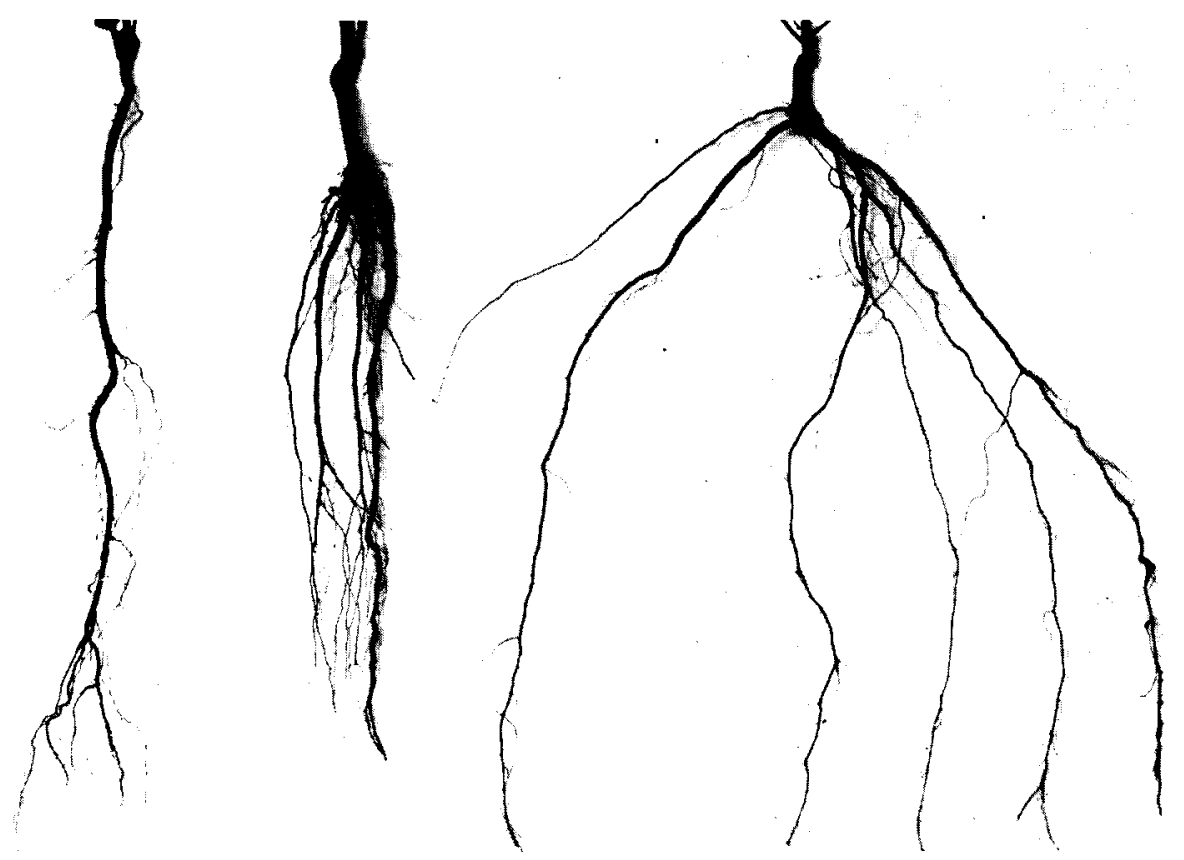

FIG. 14

Morphologie du système racinaire de semis de chêne d'un an élevé sur tourbe fertiliséé et en poubelle (un plant par poubelle).

\section{- A gauche : témoin.}

\footnotetext{
4 pivots orthogéotropes remplacent le pivot d'origine.

- A droite : pivot bloqué à $6 \mathrm{~cm}$ du collet. Des racines secondaires se sont transformées et sont devenues progressivement orthogéotropes.
}

Morphology of one year old root systems grown on fertilized peat in large containers.

- Left : control.

- Middle : taproot decapited at $6 \mathrm{~cm}$ from the collar when it was $10 \mathrm{~cm}$ long : 4 orthogeotropic taproots replace the original taproot.

- Rigtht : taproot blocked at $6 \mathrm{~cm}$ from the collar. Some secundary root became transformed and progressively orthogeotropic.

\section{Summary}

Effect of blocking the tip of the taproot on root morphogenesis of oak seedlings

Quercus robur seedlings grown in aeroponics and in minirhizotrons filled with peat behave fairly similarly (fig. 7). When the tip of $6 \mathrm{~cm}, 20 \mathrm{~cm}$ or $35 \mathrm{~cm}$ long taproots is 
blocked (see treatment 1 to 3 on fig. 1) growth of the most distal lateral roots is stimulated (fig. $2,10,11,12$ ). We call these roots «transformed secundary roots». Some continue to grow and become orthogeotropic (fig. 8, 9). When all the growing secundary roots are plastered (fig. $1 \mathrm{P}_{35}$ al) some secundary roots which were no longer growing thicken their tip and resume growth (fig. 13). «Late secundary roots» appear also amongst the former lateral roots (rlt in fig. 13). The diameter of their apex is from the beginning much greater (fig. 5). Eventually they will continue to grow and to thicken more than the regular lateral roots (fig. 6 and 14).

On root systems grown in aeroponics secundary roots may also resume extension growth for a few days after growth cessation and some «late lateral roots » may appear even on control seedlings.

The data obtained migtht be explained by taking into account :

1) Active growth correlations.

2) Differences in growing abilities of the lateral roots. These differences migth however have been determined by the complex of correlations in which they were born.

3) Variations of hormonotrophic pools available for the growth of the root system.

Reçu pour publication le 20 octobre 1982.

\section{Références bibliographiques}

Champagnat M., Baba J., Delaunay M., 1974. Corrélation entre le pivot et ses ramifications dans le système racinaire de jeunes chênes cultivés sous brouillard nutritif. Rev. Cytol. Biol. vég., 37, 407-418.

Delran S., Garbaye J., Le Tacon F., 1975. Production rapide de plants feuillus sur tourbe fertilisée. Nouveaux résultats. Rev. for. fr., 6, 436-448.

Dyanar-Najed H., 1970. Contrôle de lia plagiotropie des racines latérales chez Theobroma cacuo L. Bull. Soc. Bot. Fr. Mém., 117, 183-192.

Dyanat-Najed J. el Neville P., 1972. Etude du mode d'action du méristème radical orthogéotrope dans le contrôle de la plagiotropie des racines latérales chez Theobroma caco L. Rev. gén. Bot., 79, 319-340.

Lamond M., Mousain D., 1979. Dispositif de culture de plantes entières sur caisson à aérosol nutritif. Symposium Physiologie des racines et symbioses, I.U.F.R.O., 11-15 septembre 1978, Nancy, tome 6, p. 488-493. Ed. A. Riedacker et J. Gagnaire, C.E.N.G.I.N.R.A. Grenoble-Nancy.

LAMOND M., 1979. Influence des cotylédons sur la croissance et le développement du système racinaire du chêne pédonculé. Compte rendu du séminaire du groupe d'étude des racines, I.U.F.R.O., 11-15 septembre 1978, Nancy, tome 6, p. 228-241. Ed. A. Riedacker et J. Gagnaire, C.E.N.G.-I.N.R.A. Grenoble-Nancy.

Riedacker A, 1974. Un nouvel outil pour l'étude des racines et de la rhizosphère : le minirhizotron. Ann. Sci. for., 31, 129-134.

RIEDACKER A., 1976. Etude des possibilités de modifier la morphogénèse juvénile des systèmes racinaires de quelques espèces forestières. Applications pratiques. Proc. XVI I.U.F.R.O. World Congress, Norvège, Division 1, pp. 376-387.

Riedacker A. et Poda U., 1977. Les systèmes racinaires de jeunes plants de hêtre et de chêne. I. Modification de leur morphogénèse par décapitation d'extrémité de racines et conséquences pratiques. Ann. Sci. for., 34, 111-135.

Riedacker A., Dexheimer J., Tavakol R., Alaoui H., 1982. Modifications expérimentales de la morphogénèse et des géotropismes dans le système racinaire de jeunes chênes. Can. J. Bot., 60, 765-778. 